\title{
Rethinking Issues of Migration and Brain Drain of Health-Related Professionals: New Perspectives
}

\author{
Chux Gervase Iwu \\ Faculty of Business, Cape Peninsula University of Technology, South Africa \\ Email: iwuc@cput.ac.za
}

\section{Doi:10.5901/mjss.2014.v5n10p198}

\begin{abstract}
The intention of this study was not to particularly engage issues of health worker brain drain and migration, but to reveal some of those elements which can motivate and retain health-related professionals in South Africa. Data for this study was collected with the help of Plus Delta Organisational Climate Questionnaire. This instrument was deemed fit for the study considering its previous applications and also because it contains both job satisfaction and organisational climate elements. With the help of factor analysis, the study identified seven factors which influence employee satisfaction within the health related professions of South Africa. These factors are Role Clarification and Job Design; Equitable Performance Management; Integrated Leadership and Knowledge Sharing; Self-efficacy; Family-friendly Work Environments; Leader Credibility and Innovation and Excellent Customer Relations and Technology. The paper concludes by suggesting that these factors should be present in organisations in order to stem the tide of brain drain and migration of South African health-related professionals.
\end{abstract}

Keywords: migration, brain drain, health-related professionals, healthcare, Africa,

\section{Introduction and background}

This study responds to the call by several researchers for a sustained engagement of African health worker brain drain and migration. A sustained engagement can be in the form of academic research (Diallo, 2004; Serour, 2009; Nair \& Webster, 2013); public and private firm intervention (Serour, 2009), and or advocacy (The Partnership for Maternal, Newborn and Child Health, 2008). In this study, the author engages the subject from the perspective of health-related professionals. This band of health professionals comprises a diverse group of practitioners who deliver high quality care to patients across a wide range of care pathways and in a variety of settings. They include nurses, optometrists, radiographers, pharmacists, laboratory technologists/technicians, and emergency medical services. With diverse roles, these highly skilled professionals can be found in both the private and the public sector. They perform essential diagnostic and therapeutic roles, and work across a wide range of locations within acute, primary and community care. They perform functions, which include assessment, diagnosis, emergency care, treatment and discharge throughout the care pathway - from primary prevention through to specialist disease management and rehabilitation. Health-related professionals help to provide a well-rounded team that diagnoses and treats patients.

Nurses are generally regarded as the first point of call in health care. They not only serve the doctors who ultimately see to the medical needs of patients, but they also ensure that patients are examined properly. While Fox et al. $(1990: 227)$ refer to nurses as those who provide care for people in the midst of health, pain, loss, fear, disfigurement, death, grieving, challenge, growth, birth, and transition on an intimate front-line basis, Radiographers are the ones who perform a multitude of complex and life dependent functions, such as x-rays, fluoroscopy, computed tomography, magnetic resonance imaging, and ultrasound (Demand Media, Inc., n.d).

The other health-related professionals include pharmacists, optometrists, laboratory technicians and emergency medical service team. Pharmacists are also revered for having direct input into the prescribing process and also for helping doctors, nurses, other health professionals and especially patients, with all aspects of drug therapy. According to Millidot (2004) and Martin (2010), an Optometrist is trained to test people for spectacles and provide comprehensive eye and vision care, including refraction and dispensing, detection and diagnosis and management of eye diseases.

Laboratory technologists/technicians perform the more complex analytical processes within the laboratory. Some of their typical functions include among others, routine laboratory procedures such as preparation of media and basic preparation of slides and smears, and maintaining simple laboratory records and inventory for supplies and reagents (United Kingdom, 2010).

The goal of most emergency medical services is to either provide treatment to those in need of urgent medical care, with the goal of satisfactorily treating the malady, or arranging for timely removal of the patient to the next point of 
definitive care. Emergency medical services include ambulance services and paramedics. (U.S National Library of Medicine, n.d).

There is no doubt that the diverse group of health-related professionals such as nurses, radiographers, pharmacists, lab technologists, paramedics, and so on, share similar societal values. These practitioners are viewed as those who promote health of all people within their reach in their respective countries, by means of delivering high quality care, which is tailored to specific illnesses of their citizens.

Research indicates that in South Africa, healthcare professionals are likely to migrate to developed countries (such as the US and the UK), thus denying their nations the benefit of their training. This is generally regarded as brain drain. Serour (2009:175) however describes brain drain of health care professionals as migration from low and low/middle income countries to well developed countries; and from rural to urban areas. Essentially, the issue of health professionals' brain-drain is pervasive in sub-Saharan African countries (Connell et al., 2007:1877; Ikenwilo, 2007:3-4).

This paper takes to task socioeconomic issues of health care in Africa which include migration and brain drain (Bach, 2003; Okeke, 2013). These issues are however examined in this study within the context of health-related professionals in South Africa. The author considers these issues particularly relevant in contemporary Africa's health system because (1) Africa seems to be the home of most epidemics; (2) research has shown that a common relationship exists between the health of citizens of a nation and poverty; (3) the continuous loss of health care personnel to developed countries will result in an impoverishment of a continent and dependence on other nations; (4) perhaps the receiving nations of these health care personnel will do a rethink because continuous dependence on their economies by deprived nations can cause an implosion of their own economies.

Human resources are central to the health care system of any country and essential to the delivery of services to patients. Therefore, the positive contributions of a country's health workforce to its socio-economic challenges, as well as the economic growth are somewhat indisputable (Connell et al., 2007:1876). For instance, without a healthy workforce in any organisation, the organisation's productivity would be depressingly affected. This study thus adds to the growing literature on job satisfaction of the health workforce by providing a model of health worker satisfaction which can be applied in health establishments. As Serour (2009: 177) suggests, measures to bring about a favourable work environment among healthcare professionals need to emerge and be well implemented.

Therefore, this study took aim at (1) finding and suggesting alternative ways of reducing the high levels of attrition (caused by brain drain and migration) amongst health-related professionals, and (2) adding to the growing literature on health worker migration and brain drain.

\section{Theoretical Perspectives on Brain Drain and Migration}

\subsection{Brain drain}

Research reveals that there are lots of factors that exacerbate the brain-drain of healthcare professionals. These include economic, social, political and cultural factors, globalisation, improved communication and targeted migration (Ikenwilo, 2007:1). Ugwu et al. (2011:14) argue that high level of stress at work could also result in brain-drain, burnout and voluntary termination/resignation of employment. Brain drain of healthcare professionals may negatively affect the health care promises of a country (Ikenwilo, 2007:1). For instance, issues of the inadequacy of health care facilities and the enabling environment to retain health care personnel may emerge. Worryingly, in most cases the source countries usually have invested large amounts of monies and time in training and developing such professionals. Ikenwilo sees this as a potentially politically sensitive agenda, both for source and the destination country.

The cost of outflow of healthcare professionals can be somewhat substantial, particularly when the less-developed economies invest in educating their healthcare workers only to have them leave for wealthier nations. This could make the rich nations richer and the poor nations poorer, and a curse for economic development (Serour, 2009:176). Owing to brain drain of health workers, it becomes impracticable for the source country to achieve long-term economic growth and social development driven by talented professionals. Serour also states that sourcing expertise and capabilities from the developed countries to compensate for the brain-drain will cost the less-developed nation huge amounts of money. Brain drain weakens further, the already fragile healthcare services in less-developed countries, particularly among aspects such as maternal and neonatal health (Serour, 2009:178).

\subsection{Migration}

In developing economies, reasons for migration within the health sector have been associated with comparatively poor 
salaries, unstable governments (resulting in impoverished health facilities and fewer job opportunities for health professionals), low level of development and frustration of practising in the native country (Serour, 2009:176). Research indicates that in South Africa, nurses are generally dissatisfied with their working environment. Factors such as remuneration; poor working conditions and organizational climate were highlighted as the main contributing factors to job dissatisfaction (Pillay, 2009:2). Govender et al. (2013:61) found that the above factors were among key influential factors for migration in South Africa. They also felt that the non-existence of counter and control measures to migration of healthcare workers could exacerbate migration trends in South Africa and other African countries that experience similar conditions.

In numerous African countries young recruits including unqualified personnel in the field of healthcare, are in the likelihood of facing the challenge of being left alone to work without supervision. This exposes them to the risk of making erroneous diagnoses and inappropriate prescription of treatment to patients as a result of performing duties that are highly specialized, which also fall beyond their scope of practice (Awases et al., 2004: 58 in Connell et al., 2007:1885). Migration does not only represent negativity on the healthcare system of source countries. It also reflects in rural and urban poor communities, where a lot of people are mostly dependent on the public health systems (Connell et al., 2007:1885).

Serour (2009:177) however postulates the following measures to combat migration of the health workforce:

- Improving the health workforce database in order to keep tabs on the trends and identify possible causes.

- Improving wages, health resources, and working conditions.

- An insistence on payback from recipient countries and migrant health professionals.

- Task shifting.

- Ensuring that there is adequate investment in the health workforce.

- Up-to-date; relevant medical training and research.

Apart from brain drain and migration, for some healthcare professionals, in particular those who do not have any intentions of migrating, but experience unsatisfactory work conditions, their work performance may negatively be affected, and this perhaps may lead to regular absenteeism. Munro (2007:21) postulates that absenteeism is among prime issues that a manager has to deal with, on an on-going basis in the operation of an organisation. Sadly, in South Africa it is estimated that absenteeism costs organisations billions of rands each year (Johnson, 2007; Stokes, 2008 in Banks et al., 2012:1). Given that fewer health professionals remain to service patients, it is argued that those who are left behind are then compelled to deliver medical services to patients. This can bring about occupational stress. Rothmann and Malan (2007) confirm that occupational stress may result in impaired performance or reduced productivity, declined levels of customer service, health problems, absenteeism, low turnover, industrial accidents, substance abuse and purposefully antisocial behaviours in the organisation.

\section{Data Collection}

Data for this study was collected with the help of Plus Delta Organisational Climate Questionnaire. This instrument was deemed fit for the study considering its previous applications (De Cock, 2006; Iwu et al., 2012) and also because it contains both job satisfaction and organisational climate elements. To undertake a reliable study on the reasons for high attrition rate and high rate of absenteeism among health-related professionals, a study must investigate elements of organisational climate as well as the facets of job satisfaction (Pillay, 2009; Hinami et al., 2012; Klopper et al., 2012).

According to Plus Delta Consulting, author of the Organizational Climate Questionnaire, the instrument is an ideal tool to help management to better understand how employees think and feel as contributing members of their organizations. Plus Delta Consulting further argues that with an accurate picture of employees' attitudes, management will be able to create an action plan with specific solutions that address any areas of concern or in need of improvement. No wonder therefore, De Cock (2006) thought of the instrument as highly useful and condensed justifying its application to health-related professional's research. This band of professionals work in very high pressured environments (McCann et al., 2009: 192), thus getting them to respond to surveys is usually a tough call (Pillay, 2008:262). Therefore the researcher thoughtfully considered a single data collection instrument as opposed to a multitude of instruments that may result in loss of valuable time for the health-related personnel. After all, questionnaires are known to possess the attractive quality of time saving especially given that the researcher or interviewer is not present to interrogate the responses.

Usable data came from only 117 health-related practitioners (47 Nurses; 26 Optometrists; 24 Paramedic personnel; 7 pharmacists; and 13 Radiographers) practising in both private and public health establishments within Western Province of South Africa. 
The analysis of the data was conducted with the help of factor analysis. The justification for factor analysis as the most suitable statistical technique for this study was based on its ability to simplify complex sets of data. The instrument utilised for data collection in this study had 11 categories and 53 items. It would have been disingenuous to utilise another approach to analyse data besides factor analysis. This is because factor analysis has the capacity to discover the underlying patterns or relationships in a large number of variables and can reduce these variables to a smaller set of factors or new variates (Blaikie, 2003: 155), and also referring to Leong and Austin (2006: 250), variables (or items) that are generally quite small, are unlikely to give rise to sensible common factors.

Zikmund et al. (2010: 593) provide support for the researcher's submission by adding that factor analysis is a technique that statistically identifies a reduced number of factors from a larger number of measured variables. According to them, the factors themselves are not measured, but are identified by forming a variate using the measured variables. Kline (2002:1) regards factor analysis as the best statistical technique for psychological studies as well as the social sciences. Another reason why factor analysis was chosen for this study was because of its exploratory nature (Zikmund et al., 2010: 593), which supports the nature of this study - an exploratory study that wished to develop a model of health worker satisfaction within the health related professions of South Africa because no model of this nature currently exists within the health related professions of South Africa.

\section{Findings}

Utilizing factor analysis, this study was able to identify seven (7) factors which influence employee satisfaction within the health related professions of South Africa. These factors include Role Clarification and Job Design; Equitable Performance Management; Integrated Leadership and Knowledge Sharing. The others include Self-efficacy; Familyfriendly Work Environments; Leader Credibility and Innovation and Excellent Customer Relations and Technology. Each of these factors has emerged from a mix of categories (constructs) in the Plus Delta Organisational Climate Questionnaire. These factors now form part of the model which is shown below (Figure 1). The demographic properties (namely income, tenure, race, marital status and age) which have high statistical significance to the factors are also contained in the model.

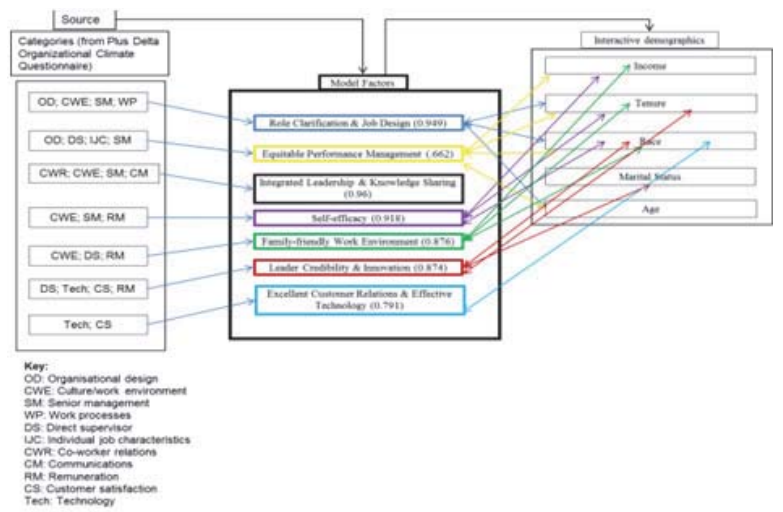

Figure 1: Model of Employee Satisfaction for health-related professions

\subsection{The model explained}

The factor - role clarification and job design - was derived from a mix of categories, namely organizational design (OD), culture/work environment (CWE), senior management (SM) and work processes (WP). With a high cronbach alpha of .949 , the factor interacted well with tenure, race and age.

Equitable performance management was derived from the categories organizational design (OD), direct supervisor (DS), individual job characteristics (IJC) and senior management (SM). Achieving a cronbach alpha of .662, the factor shared high statistical relationship to income, tenure, race and age.

The categories from which the factor -Integrated leadership and knowledge sharing - emerged include co-worker relations CWR), culture/work environment (CWE), senior management (SM) and communication (CM). The items were brought together because of their high correlations with each other, thus indicating a significant and positive relationship among the items. Together, the items achieved a high cronbach alpha of 0.96 .

With regard to Self-efficacy, the items in this factor were derived from two major organizational climate dimensions (culture/work environment (CWE), and senior management (SM) and one job satisfaction facet, namely remuneration $(\mathrm{RM})$ ). They have been grouped together under the factor self-efficacy because of their high factor loadings. Together, 
these items achieved a cronbach a of 0.918 . As the figure above shows, the factor self-efficacy interacted positively with income, tenure and race.

Family-friendly work environment (FFWE) was derived from the categories culture/work environment (CWE), direct supervisor (DS) and remuneration (RM) because of their significant and positive statistical relationship. The items together achieved a significantly high cronbach coefficient alpha of 0.86 . Family-friendly work environment interacted well with the following demographic properties: race, tenure and income.

The model factor named Leader credibility and innovation was derived from the categories direct supervisor (DS), technology (Tech), customer satisfaction (CS) and remuneration (RM).Together, the items achieved a high cronbach alpha of 0.874 .this factor shared close relationship with marital status, race and tenure.

Excellent customer relations and effective technology emerged from the categories technology (Tech) and customer satisfaction (CS) because the items had high significant factor loadings with a strong cronbach alpha of 0.791 . This factor had high statistical association to race.

Empirical findings from this study indicate that role clarity and job design were critical in explaining reasons for this study's objectives. Previous studies provide eminent support in this regard. For instance Albion et al. (2008: 279) warned that role clarity was the only organisational climate variable that emerged in their study as a unique contributor to the formation of intentions to leave the health profession. There was also an urgent call by Rowe et al. (2005) for health care establishments to improve role clarity. A well-designed job improves the psychological meaningfulness of a role and is, according to Bakker and Demerouti (2007. Cited in Xu \& Thomas, 2010: 401), associated with high work standards. Tenure, race and age were the demographic variables that interacted positively with this factor.

It was also the contention of this study that equitable performance management would prevent health-related professionals from looking elsewhere (outside the country or in other sectors) for greener pastures. Health-related personnel would feel very comfortable in an environment where both senior management and direct supervisor show appreciation for one's work either through the provision of helpful feedback, or through well-defined structures with policies, procedures and systems that allow employees to achieve personal and organizational goals. As figure one above shows, this factor had very positive relationship with income, tenure, race and age.

The factor - Integrated Leadership and Knowledge Sharing - typifies a work environment where senior management encourages collaboration across all levels; where individuals appreciate the personal contribution of their peers (without any form of negative attitude); and where individuals are consulted to take part in matters that affect them.

The factor - Self-efficacy- draws inspiration from the argument that health-related personnel function better if they have very high sense of worthiness in and or belief in their capabilities to manage responsibilities. This will come from the health-related personnel feeling valued as an employee; receiving meaningful remuneration and feeling that management treats him fairly. Employers of health-related personnel must be able to show care, interest and empathy with employees. They should also be able to explain what is expected of the health-related professionals as well as provide regular and positive feedback and recognition for work well done. Income, tenure and race were the demographic variables that had significant relationship with this factor.

There is an abundance of empirical evidence that family responsive workplaces reduce absenteeism, intent to leave the organization as well as stress and conflict. Family-friendly work environments are characterized by flexible work arrangements and child-care and exercise facilities. They are essentially a more fluid environment where the healthrelated professional experiences a sense of community. Family-friendly work environment was found to have very positive relationship with income, tenure and race.

Leader credibility and innovation extends the thinking of previous researchers who recommended transformational leadership, and provision of quality service using adequate technology. This factor gives impetus to the model of employee satisfaction because it recognizes the influence of poor technology on the quality of service delivery. This factor also argues that the ability of the leader to give helpful feedback also helps to generate some confidence in the employee that he is dealing with a competent superior. It therefore behoves the superior to act in a manner that lends trust in his competence to lead. To gain the trust of employees, managers must be seen to be knowledgeable in their work, caring of employees and approachable. The consequence of credible leadership is that of reduced tension and better juggling of work roles. This factor showed very close association with tenure, race, and marital status.

Quality service enriches market share. If external customers are satisfied, internal customers (in this case, employees) will feel a sense of self-worth - a feeling that they contribute to the growth of the organization. Customers then become recipients of good service. To achieve world class excellence in service and also meet and possibly exceed customer's expectations, health care establishments must therefore understand the specific needs of their customers. To do this however requires functional tools and technologies that support the health-related employee in completing his work. Health-related establishments are technology driven and as a result they must have technology that supports their 
business processes. The factor - Excellent Customer Relations and Effective Technology - argues in support of a model of employee satisfaction within the health-related professions of South Africa. Race was the only demographic variable found to have a positive relationship with this factor.

Reporting Kanter's (1994) findings, Lee and Teo (2005: 28) submitted that positive organizational behaviour is supported by work environments that provide access to information, resources, and the opportunity to learn and develop. Essentially, Lee and Teo are saying that supervisory receptiveness to employee problems is a strong driver of employee satisfaction.

\section{Conclusion and Recommendations}

The intention of this paper was not to particularly engage issues of health worker brain drain and migration, but to reveal some of those elements which can motivate and retain health-related professionals in South Africa. South Africa presents a significant evidence of health worker brain drain and migration (Okeke, 2013) and with that many scholarly works have been undertaken to explain why (the push and pull factors) South African health workers are more susceptible to migrate to developed countries. A major contribution of this paper therefore is the introduction of a new model that can help stem the tide on health worker brain drain and migration; especially health-related professionals. Using factor analysis, the author has been able to identify seven factors which need to be in place for these health-related professionals to keep their jobs. As Ikenwilo (2007: 3) noted; a job-scarce environment as well as underemployment could lead to brain drain and migration. This assertion can be linked to Iwu et al's (2013) findings, which postulated that there is a link between job satisfaction and work-overload. In the event that health-related professionals leave their jobs or their nations in search of greener pastures, the remaining staff will be pressured to deal with the heavy workload. This may lead to stress and low self-confidence, which may ultimately push some healthcare professionals to migrate (Ikenwilo, 2007:3). When the pressures of attending to the health of clients are curtailed, it is expected that health-related professionals will provide much stronger support to their organizations. If an organization's climate is unfavourable, it is likely to influence the health-related professional into having several negative influences, which might plague his total work commitment.

It is therefore critical that health establishments and governments acknowledge the above facts. A strategic approach that will discourage healthcare professionals from exiting the country needs to emerge. For instance, a favourable working environment, which can provide all or some of the factors captured in the model to healthcare workers, would enhance chances of these individuals to remain in their country of origin. For instance health-related personnel must be integrated into matters that affect them. Integrated leadership can be empowering. When employees are involved in matters that affect them, they feel valued, respected and they consequently value their membership of an organization. Formal and informal face-face meetings and socialization opportunities can enhance trust and communication among units. Achieving trust requires an environment that acknowledges the informal organization - the network of relationships that facilitate the actioning of the responsibilities.

It is also critical that health-related professionals are made aware of what is expected of them through organized communication systems. They produce better when they are clear on how best to perform their tasks especially when their tasks have high psychological meaningfulness. There is also a need for family-friendly work environments. Flexible working hours, child-care utilities as well recreational facilities can take away the pain from the long arduous schedule of the health-related professional. In this case, they are then able to relax and also find time to spend with their families. Recreational facilities help with socialization at work.

Performance management systems must also not only be fair but should be seen to be so. This is in order to achieve high employee morale and satisfaction among health-related personnel who need to feel a sense of value both in them and in their jobs. The feeling of self-worth comes with comparative remuneration, a sense of fair treatment from management and the perception that their efforts are significantly related to organizational goals. This will help reduce the high incidences of brain-drain, migration and absenteeism.

While the author feels that this paper makes an important contribution to the growing literature on health worker migration and the brain drain, the author acknowledges that there are certain limitations. An obvious limitation is the one around generalization. While South Africa presents a classical example in terms of large numbers of health worker migration and brain drain, this study only focused on a province in South Africa - Western Province. Therefore, generalization of the model presented here should be made with caution. The use of a questionnaire may have prevented helpful responses from coming through. For instance, if an interviewer was present to ask for clarification to some of the responses, perhaps a much clearer perspective may have been realized from the health-related professionals sampled. Another limitation of the study is that the sample had more nurses $(n=47)$ perhaps because they constitute the largest number of health workers that felt the need to participate in the study. Future studies may look at maintaining a balance between the health-related professionals in order to determine whether there will be significant changes in their results. 
The author further advises that perhaps exploratory factor analysis may not have been sufficient in achieving the nearest clusters of factors. To this end, the author invites researchers to explore the possibility of further interrogation of the model and its factors in employee/job satisfaction studies as this may help to provide deeper answers to management scholarship in the realm of health worker migration, brain drain and employee/job satisfaction. This will no doubt advance the call made by Bach (2003) that governments and employers should do more to stem the tide of migration and brain drain.

\section{References}

Albion, M.J., Fogarty, G.J., Machin, M.A., \& Patrick, J. (2008). Predicting absenteeism and turnover intentions in the health professions. Australian Health Review, 32(2), 271-281.

Awases, M. Gbary, A., Nyoni, J., \& Chatora, R. (2004). Migration of health professionals in six countries. Brazzaville: WHO Regional Office for Africa.

Bach, S. (2003). International migration of health workers: Labour and social issues. Geneva: International Labour Office.

Banks, J. Patel, C. J. \& Moola, M. A. (2012). Perceptions of inequity in the workplace: Exploring the link with unauthorised absenteeism. South African Journal of Human Resource Management, 10(1), 1-8.

Blaikie, N. (2003). Analyzing quantitative data. From description to explanation. London: SAGE.

Connell, J., Zurn, P., Stilwell, B., Awases, M., \& Braichet, J.M. (2007). Sub-Saharan Africa: Beyond the health worker migration crisis? Social Science \& Medicine, 64(9), 1876-1891.

De Cock, C. (2006). A Creativity Model for the Analysis of Continuous Improvement Programmes: A Suggestion to Make Continuous Improvement Continuous. Creativity and Innovation Management, 2(3): 156-165.

Demand Media. Inc. (n.d). Role of a radiographer. Retrieved from http://www.ehow.com/about_5087423_role-radiographer.html. March 24, 2010

Diallo, K. (2004). Data on the migration of health-care workers: sources, uses, and challenges. Bulletin of the World Health Organization, 82(8), 601-607.

Fox R.C., Aiken, L.H., \& Messikomer, C.M. (1990). The Culture of Caring: AIDS and the Nursing Profession. Milbank Quarterly., 68, Supplement 2 (Part 2):226-256.

Govender, K. Grainger, L. \& Naidoo, R. (2013). Developing retention and return strategies for South African advanced life support paramedics: A qualitative study. African Journal of Emergency Medicine, 3, 59-66.

Hinami, K., Whelan, C. T., Wolosin, R. J., Miller, J. A., \& Wetterneck, T. B. (2012). Worklife and satisfaction of hospitalists: toward flourishing careers. Journal of General Internal Medicine, 27(1), 28-36.

Ikenwilo, D. (2007). Brain Drain: Painting a Picture for Africa. Nairobi: African Technology Policy Studies Network.

Iwu, C, G., Gwija, S.A., Benedict, H. O., \& Tengeh, R. K. (2013). Teacher job satisfaction and learner performance in South Africa. Journal of Economics and Behavioral Studies, 5 (12), 2220-6140.

Iwu, C. G. Allen-lle, C. \& Ukpere, W. I. (2012). A model of employee satisfaction amongst health-related professionals in South Africa: The case of Western Cape Province. African Journal of Business Management, 6 (34), 9658-9670, August.

Johnson, J. (2007). Absenteeism trends in South African companies - The biggest cause of absenteeism. Human Capital Review. http:/l uww.humancapitalreview.org/content/default [26 March 2011].

Kingma, M. (2007). Nurses on the Move: A Global Overview. Health Services Research, 1281-1298.

Kline, P. (1994). An easy guide to factor analysis. London: Routledge.

Klopper, H. C., Coetzee, S. K., Pretorius, R., \& Bester, P. (2012). Practice environment, job satisfaction and burnout of critical care nurses in South Africa. Journal of Nursing Management, 20(5), 685-695.

Lee, G., \& Teo, A. (2005). Organizational restructuring: Impact on trust and work satisfaction. Asia Pacific Journal of Management, 22, 23-39.

Leong, F.T.L., \& Austin, J.T. (Eds.). (2006). The Psychology Research Handbook. A Guide for Graduate Students and Research Assistants. (2nd ed.). London: SAGE.

Martin, E. A. (Ed.). (2010). Concise Medical Dictionary. Oxford University Press.

McCann, L., Hughes, C.M., Adair, C.G., \& Cardwell, C. (2009). Assessing job satisfaction and stress among pharmacists in Northern Ireland. Pharmacy World \& Science, 31, 188-194.

Millidot, M. (2004). Dictionary of Optometry and Visual Science. (6 $6^{\text {th }}$ ed.). China: Butterworth-Heinemann

Munro, L. (2007). Absenteeism and presenteeism: possible causes and solutions. The South African Radiographer, 45(1), 21-23, April.

Nair, M., \& Webster, P. (2013). Health professionals' migration in emerging market economies: patterns, causes and possible solutions. Journal of Public Health, 35(1), 157-163.

Okeke, E. N. (2013). Brain Drain. Social Science and Medicine, 93, 169-178.

Pillay, R. (2008). Work satisfaction of medical doctors in the South African private health sector. Journal of Health Organization and Management, 22(3), 254-268.

Pillay, R. (2009). Work satisfaction of professional nurses in South Africa: a comparative analysis of the public and private sectors. Human Resources for Health, 7(15), 110.

Rothmann, S., \& Malan, M. (2007). Occupational stress of hospital pharmacists in South Africa. The International Journal of Pharmacy Practice, 235-242, September.

Rowe, A.K., de Savigny, D., Lanata, C.F., \& Victoria, C.G. (2005). How can we achieve and maintain high-quality performance of health workers in low-resource settings? Lancet, 366, 1026- 1035.

Serour, G. I. (2009). Healthcare workers and the brain drain. International Journal of Gynaecology \& Obstetrics, 106(2), 175-178.

South Africa National Treasury. (2008). Estimates of national expenditure. Pretoria: National Treasury.

Stokes, G. (2008). Managing sick leave. http://www. fanews.co.za/article.asp?Front_Page_Features;25;Straight_Talk;1146,Managing_sick_leave [18 June 2010].

The Partnership for Maternal, Newborn and Child Health. The Partnership Update - March 2008. Available at: http://www.who.int/pmnch/media/publications 1200803update/en/index.html.

Ugwu, A. C., Erondu, O. F., \& Umeano, U. B. (2011). Psychosocial stress and its predictors among radiographers in south-eastern Nigeria. The South African Radiographer, 49(2), 11-15.

United Kingdom. (2010), September. Scientific laboratory technician. Job description. Retrieved from http://www.prospects.ac.uk/scientific_laboratory technician_job_description.htm. April 6, 2012.

U.S National Library of Medicine. (nd). Emergency Medical Services. Retrieved from http://www.nlm.nih.gov/medlineplus/emergencymedicalservices.html March 10 2014.

Xu, J., \& Thomas, H.C. (2010). How can leaders achieve high employee engagement? Leadership \& Organization Development Journal, 32(4), $399-416$.

Zikmund, W.G., Babin, B.J., Carr, J.C., \& Griffin, M. (2010). Business Research Methods. (8th ed.). Canada: South-Western. 